UDC 517.53

\author{
V. I. Danchenko, D. G. Chkalova
}

\title{
BERNSTEIN-TYPE ESTIMATES FOR THE DERIVATIVES OF TRIGONOMETRIC POLYNOMIALS
}

\begin{abstract}
Using the method of amplitude and phase transformations, we obtain sharp inequalities for the derivatives of real-valued trigonometric polynomials. The inequalities are sharp, as there are the corresponding extremal polynomials, for which they become equalities.
\end{abstract}

Key words: amplitude and phase transformations, Bernstein's inequality

2020 Mathematical Subject Classification: 26D05

1. Introduction. For a positive integer $n$, set

$$
T_{n}(t)=\sum_{k=0}^{n} \tau_{k}(t), \quad \tau_{k}(t):=a_{k} \cos k t+b_{k} \sin k t, \quad t, a_{k}, b_{k} \in \mathbb{R} .
$$

An amplitude and phase transformations (AFT) of order at most $n$ is the sum

$$
H\left(T_{n},\left\{X_{j}\right\},\left\{\lambda_{j}\right\} ; t\right):=\sum_{j=1}^{n} X_{j} T_{n}\left(t-\lambda_{j}\right),
$$

where $\lambda_{j}, X_{j}$ are free real parameters (some $X_{j}$ may be zero). The order of an AFT is equal to the number of summands in $H\left(T_{n},\left\{X_{j}\right\},\left\{\lambda_{j}\right\} ; t\right)$ with pairwise-distinct $\exp \left(i \lambda_{j}\right)$ and $X_{j} \neq 0$. The AFTs were introduced and used in the papers [7], [18], [19] for obtaining Fejer-type estimates for harmonics and coefficients of trigonometric polynomials. Estimates of this type are well-known in extremal problems for nonnegative trigonometric polynomials (see, e.g., [1], [3], [8], [10], [14], [16]). Algebraic analogues of the AFTs, the so-called amplitude and frequency operators, were used for the Padè interpolation in [5].

(C) Petrozavodsk State University, 2021 
Denote by $w$ a set of real weights $w:=\left\{\sigma_{1}, \sigma_{2}, \ldots, \sigma_{n}\right\}, \sigma_{l} \in \mathbb{R}$, and determine the sums of weighted harmonics of the form

$$
\mathcal{T}\left(T_{n}, w ; t\right):=\sum_{\sigma_{l} \in w} \sigma_{l} \tau_{l}(t) .
$$

Let us consider the problem of representing the weighted sums in the form of AFTs:

$$
\omega a_{0}+\mathcal{T}\left(T_{n}, w ; t\right)=H\left(T_{n},\left\{X_{j}\right\},\left\{\lambda_{j}\right\} ; t\right),
$$

where $X_{j}, \lambda_{j}, \omega$ are the required real parameters and $a_{0}=a_{0}\left(T_{n}\right)$ is the constant term of the polynomial $T_{n}$. In this case, the equality $\omega=\sum_{j=1}^{n} X_{j}$ must naturally hold. It is easy to show that the problem (2) is equivalent to the following discrete moments problem:

$$
X_{1} z_{1}^{l}+\ldots+X_{n} z_{n}^{l}=\sigma_{l}, \quad l=\overline{1, n}, \quad z_{j}=e^{-i \lambda_{j}},
$$

with real unknowns $X_{j}$ and $\lambda_{j}$, where some $X_{j}$ may take zero values, and $z_{j}=e^{-i \lambda_{j}}$ are distinct. Indeed, given a real-valued $T_{n}$, we have $\tau_{k}(t)=2 \cdot \operatorname{Re}\left(\alpha_{k} e^{i k t}\right)$, where $2 \alpha_{k}=a_{k}-i b_{k}$ and, therefore,

$$
\begin{array}{r}
H\left(T_{n},\left\{X_{j}\right\},\left\{\lambda_{j}\right\} ; t\right)=a_{0} \omega+2 \operatorname{Re}\left(\sum_{k=1}^{n}\left(\sum_{j=1}^{n} X_{j} z_{j}^{k}\right) \alpha_{k} e^{i k t}\right)= \\
=a_{0} \omega+\sum_{k=1}^{n} s_{k} \tau_{k}(t),
\end{array}
$$

where

$$
\omega:=\sum_{j=1}^{n} X_{j}, \quad s_{k}:=\sum_{j=1}^{n} X_{j} z_{j}^{k}, \quad s_{k} \in \mathbb{R} .
$$

Hence, when (3) holds, (4) gives (2). The converse is also true: from the equalities (2) and (4) we obtain (3) (for more details, see [7]).

According to the classical Carathèodory theorem (see, e.g., [2], [11], [13]), the system (3) always has a (unique) solution, such that $X_{j} \geqslant 0$, $z_{j}=e^{i \lambda_{k}}, \lambda_{j} \in \mathbb{R}$ (for arbitrary fixed right-hand sides $\sigma_{l} \in \mathbb{C}$ ). It follows from the uniqueness of solution, in particular, that in the case under consideration, where the right-hand sides $\sigma_{l}$ are real, the roots $z_{j}$ lie on the unit circle symmetrically with respect to $\mathbb{R}$.

An important role during the analysis of systems (3) is played by the Toeplitz matrix $G_{n+1}(w ; \omega)=\left\{g_{i, j}\right\}$ of order $n+1$ with the following 
structure: the main diagonal contains the parameter $\omega=\sum_{j=1}^{n} X_{j}$, and the weights $\sigma_{l}$ are symmetrically located on the parallel diagonals, and $g_{i, j}=\sigma_{l}$ for $|i-j|=l, l=1, \ldots, n$ (see, e. g., [19]).

It is known (see, e.g., [2], [11], [13]) that for the solution to the Carathèodory problem (i.e., the problem (3) for $X_{j} \geqslant 0$ and real $\left\{\lambda_{j}\right\}$ ), the value of parameter $\omega=\sum_{j=1}^{n} X_{j}$ is equal to the largest root $\omega_{w}^{+}$ $\left(\omega_{w}^{+}=\omega_{w}^{+}(n)>0\right)$ of the polynomial $\operatorname{det} G_{n+1}(w ; \omega)$, the determinant of the Toeplitz matrix. It obviously implies that for the solution of the problem (3) with non-positive $\left\{X_{j}\right\}$ and real $\left\{\lambda_{j}\right\}$, the value $\omega=\sum_{j=1}^{n} X_{j}$ is equal to the smallest root $\omega_{w}^{-}\left(\omega_{w}^{-}=\omega_{w}^{-}(n)<0\right)$ of the same Toeplitz matrix determinant $G_{n+1}(w ; \omega)$ (it is sufficient to consider the problem (3) with $X_{j}$ replaced by $X_{j}^{\prime}=-X_{j}$ and $\sigma_{l}$ by $\sigma_{l}^{\prime}=-\sigma_{l}$ ).

Consequently, the equalities (2) are valid for $\omega=\omega_{w}^{+}>0$ (and then all $X_{j} \geqslant 0$ ) and for $\omega=\omega_{w}^{-}<0$ (and then all $X_{j} \leqslant 0$ ). The representation (2) in each of these cases we call regular if the order of the corresponding AFT is exactly $n$ (then all $X_{j} \neq 0$ ).

If $\omega=\omega_{w}^{ \pm}$are known, the Prony polynomial $P_{n}\left(z ; w, \omega_{w}^{ \pm}\right)$is used to determine the unknowns $z_{j}=e^{-i \lambda_{j}}$; it is the determinant obtained from the above-mentioned Toeplitz determinant by replacing its first row with a row $\left(z^{n}, z^{n-1}, \ldots, 1\right)$ (see the examples below).

In a regular case, all roots $z_{j}=e^{-i \lambda_{j}}, j=1, \ldots, n$, of the polynomial $P_{n}\left(z ; w, \omega_{w}^{ \pm}\right)$are distinct and lie on the unit circle symmetrically with respect to $\mathbb{R}$. Their arguments give the required values $\lambda_{j}$ in (2) and (3). In this case, the unknowns $X_{j}$ are found from the system (3), linear with respect to $X_{j}$ with a nonzero Vandermonde determinant (for more details, see, e.g., [7], [18], [19]).

In an irregular case, when the order of AFT in (2) is less than $n$, the Prony polynomial is identically zero, $P_{n}\left(z ; w, \omega_{w}^{ \pm}\right) \equiv 0$. For this case, several methods have been developed to regularize the problem (3) by certain variations of the right-hand sides $\left\{\sigma_{k}\right\}$ ( [18], [19]). For the regularized system (3), the corresponding Prony polynomial is nonzero and its nonzero roots are the desired solution to the system $(3) z_{j}=e^{-i \lambda_{j}}, j=1, \ldots, m$, $m<n$.

2. Extremal polynomials in the representation (2). Let us briefly describe a method of construction extremal nonnegative polynomials $S_{ \pm}$for which the following equalities hold (for more details, see [18], [19]):

$$
\omega_{w}^{ \pm} a_{0}\left(S_{ \pm}\right)+\mathcal{T}\left(S_{ \pm}, w ; 0\right)=0, \quad w:=\left\{\sigma_{1}, \ldots, \sigma_{n}\right\}
$$


Let the representation (2) (regular or irregular) be obtained, for a given set of real weights $w:=\left\{\sigma_{1}, \ldots, \sigma_{n}\right\}$, in each of the cases $\omega=\omega_{w}^{ \pm}$, where the AFT has order $m^{ \pm} \leqslant n$. Using the obtained solutions $\lambda_{j}^{ \pm}, j=1, \ldots, m^{ \pm}$, we define the following even non-negative polynomials of order $m^{ \pm}$:

$$
S_{ \pm}(t)=S_{ \pm}\left(\omega_{w}^{ \pm} ; t\right):=\prod_{j=1}^{m^{ \pm}} \sin ^{2}\left(\frac{t+\lambda_{j}^{ \pm}}{2}\right) .
$$

Note that polynomials of the form (6) have already been used in [6], [12], [19]. Since $S_{ \pm}\left(-\lambda_{j}^{ \pm}\right)=0$ for all $j=1, \ldots, m^{ \pm}$, we have equality $H\left(S_{ \pm},\left\{X_{j}^{ \pm}\right\},\left\{\lambda_{j}^{ \pm}\right\} ; 0\right)=0$ and, therefore, we obtain (5) from (2).

This equality will be used below to prove the sharpness of Bernsteintype inequalities. Note that the parity of polynomials $S_{m^{ \pm}}\left(\omega_{w}^{ \pm} ; t\right)$ obviously follows from the symmetry of roots $z_{j}^{ \pm}=e^{-i \lambda_{j}^{ \pm}}$in (3) with respect to $\mathbb{R}$.

\section{The application of AFT for trigonometric Bernstein-type} inequalities. Let us take a set of weights $w=w(n, 2 m)$ for positive integer $n$ and $m: w(n, 2 m):=\left\{1^{2 m}, 2^{2 m}, \ldots, n^{2 m}\right\}$. For the derivative of order $2 m$ of the polynomial (1), we have

$$
(-1)^{m} T_{n}^{(2 m)}(t)=\sum_{k=1}^{n} k^{2 m} \tau_{k}(t)=\mathcal{T}\left(T_{n}, w ; t\right) .
$$

Therefore, for the largest and smallest roots $\omega_{w}^{ \pm}=\omega_{w(n, 2 m)}^{ \pm}$of the Toeplitz determinant $\operatorname{det} G_{n+1}(w ; \omega)$ from (2), we get

$$
\begin{aligned}
\omega_{w}^{ \pm} a_{0}+(-1)^{m} T_{n}^{(2 m)}(t)= & \omega_{w}^{ \pm} a_{0}+\mathcal{T}\left(T_{n}, w ; t\right)=\sum_{j=1}^{n} X_{j}^{ \pm} T_{n}\left(t-\lambda_{j}^{ \pm}\right), \\
& \sum_{j=1}^{n} X_{j}^{ \pm}=\omega_{w}^{ \pm},
\end{aligned}
$$

where $\omega_{w}^{+}>0, \omega_{w}^{-}<0$, all $X_{j}^{+} \geqslant 0, X_{j}^{-} \leqslant 0, \lambda_{j}^{ \pm} \in \mathbb{R}$.

Theorem 1. For positive integer $n, m$, weights $w=w(n, 2 m):=\left\{1^{2 m}\right.$, $\left.2^{2 m}, \ldots, n^{2 m}\right\}, a_{0}=a_{0}\left(T_{n}\right)$, and all $t \in \mathbb{R}$, the following two-sided inequalities hold:

$$
\omega_{w}^{+}\left(\min _{x} T_{n}(x)-a_{0}\right) \leqslant(-1)^{m} T_{n}^{(2 m)}(t) \leqslant \omega_{w}^{+}\left(\max _{x} T_{n}(x)-a_{0}\right),
$$




$$
\omega_{w}^{-}\left(\max _{x} T_{n}(x)-a_{0}\right) \leqslant(-1)^{m} T_{n}^{(2 m)}(t) \leqslant \omega_{w}^{-}\left(\min _{x} T_{n}(x)-a_{0}\right) .
$$

In particular, if $T_{n}$ is a positive-valued polynomial, then the first inequality in (8) and the second inequality in (9) imply

$$
-\omega_{w}^{+} a_{0} \leqslant(-1)^{m} T_{n}^{(2 m)}(t) \leqslant-\omega_{w}^{-} a_{0}, \quad a_{0}>0 .
$$

The latter two-sided inequality is sharp, as there are extremal nonnegative polynomials $S_{ \pm}(t), \operatorname{deg} S_{ \pm} \leqslant n$, for which the following equalities hold:

$$
\omega_{w}^{ \pm} a_{0}^{ \pm}\left(S_{ \pm}\right)+(-1)^{m} S_{ \pm}^{(2 m)}(0)=0, \quad a_{0}^{ \pm}\left(S_{ \pm}\right)>0 .
$$

Proof. The upper estimate in (8) is obtained from (7) taking into account that all $X_{j}^{+} \geqslant 0$ and $\omega_{w}^{+}=\sum_{j=1}^{n} X_{j}^{+}>0$ :

$$
\omega_{w}^{+} a_{0}+(-1)^{m} T_{n}^{(2 m)}(t)=\sum_{j=1}^{n} X_{j}^{+} T_{n}\left(t-\lambda_{j}^{+}\right) \leqslant \omega_{w}^{+} \max _{x} T_{n}(x) ;
$$

similarly, we obtain the lower estimate in (8). The lower estimate (9) is obtained from (7) taking into account that all $X_{j}^{-} \leqslant 0$ and $\omega_{w}^{-}=\sum_{j=1}^{n} X_{j}^{-}<0$ :

$$
-\omega_{w}^{-} a_{0}+(-1)^{m+1} T_{n}^{(2 m)}(t)=\sum_{j=1}^{n}\left(-X_{j}^{-}\right) T_{n}\left(t-\lambda_{j}^{-}\right) \leqslant-\omega_{w}^{-} \max _{x} T_{n}(x) ;
$$

similarly, we obtain the upper estimate in (9). The statement (10) follows from (5).

Examples. Let us give examples of constructing several extremal polynomials in (10) for the second derivative.

For $n=2, m=1$ and weights $w=w(2,2)=\left\{1^{2}, 2^{2}\right\}$ we have

$$
G_{3}(w, \omega)=\left[\begin{array}{ccc}
\omega & 1 & 4 \\
1 & \omega & 1 \\
4 & 1 & \omega
\end{array}\right], \quad P_{2}(z ; w, \omega)=\left[\begin{array}{ccc}
z^{2} & z & 1 \\
1 & \omega & 1 \\
4 & 1 & \omega
\end{array}\right],
$$

where $\operatorname{det} G_{3}(w, \omega)=(\omega-4)\left(\omega^{2}+4 \omega-2\right)$, and for the second derivatives the representations (7) and the inequalities (8), (9) are obtained with the parameters $\omega_{w}^{+}=4, \omega_{w}^{-}=-2-\sqrt{6} \approx-4.449 \ldots$ (here and everywhere below, numerical values are displayed with three decimal places). 
Thus, we get the two regular cases $\omega=\omega^{ \pm}$; the Prony polynomials $P_{2}\left(z ; w, \omega_{w}^{ \pm}\right)$have the pairs of roots \pm 1 and $-0.224 \pm 0.974 i$ with the arguments $\{0, \pi\}$ and $\{1.797,-1.797\}$. Hence, according to the formula (6), we find an extremal nonnegative polynomials, for which the equalities (10) hold for the second derivative:

$$
\begin{gathered}
S_{+}(t) \approx 0.125-0.125 \cos (2 t), \quad \omega_{w}^{+}=4 ; \\
S_{-}(t) \approx 0.137+0.125 \cos (2 t)+0.112 \cos (t), \quad \omega_{w}^{-} \approx-4.449 .
\end{gathered}
$$

When $n=3$ and $w=w(3,2)=\left\{1^{2}, 2^{2}, 3^{2}\right\}$, according to the same scheme, we have

$$
G_{4}(w, \omega)=\left(\begin{array}{cccc}
\omega & 1 & 4 & 9 \\
1 & \omega & 1 & 4 \\
4 & 1 & \omega & 1 \\
9 & 4 & 1 & \omega
\end{array}\right), \quad P_{3}(z ; w, \omega)=\left|\begin{array}{cccc}
z^{3} & z^{2} & z & 1 \\
1 & \omega & 1 & 4 \\
4 & 1 & \omega & 1 \\
9 & 4 & 1 & \omega
\end{array}\right|
$$

therefore, $\operatorname{det} G_{4}(w, \omega)=\omega(\omega-10)\left(\omega^{2}+10 \omega-16\right)$ and $\omega_{w}^{+}=10$, $\omega_{w}^{-}=-5-\sqrt{41} \approx-11.403$. This implies representations (7) and inequalities (8), (9). Here, for $\omega=\omega^{ \pm}$, regular cases are obtained and the Prony polynomials $P_{3}\left(z ; w, \omega_{w}^{ \pm}\right)$have three different roots with unit moduli:

$$
\begin{gathered}
\{1,-0.666-0.745 i,-0.666+0.745 i\} \\
\{-1,0.259-0.965 i, 0.259+0.965 i\} .
\end{gathered}
$$

Calculating their arguments $\lambda_{j}^{ \pm}$, by the formula (6), we find extremal nonnegative polynomials, for which the equalities (10) hold for $m=1$ :

$$
\begin{gathered}
S_{+}(t) \approx 0.034-0.031 \cos (3 t)-0.020 \cos (2 t)+0.017 \cos (t) \\
S_{-}(t) \approx 0.038+0.031 \cos (3 t)+0.030 \cos (2 t)+0.037 \cos (t) ; \\
\omega_{w}^{+}=10, \quad \omega_{w}^{-} \approx-11.403 .
\end{gathered}
$$

Remark 1. One can see from Theorem 1 that the main role is played by the module estimates $\omega_{w(n, 2 m)}^{ \pm}$for the derivative estimates based on (7). They can be estimated using the well-known Hadamard theorem about the non-degeneracy of matrices with strictly dominant diagonal (see, e.g., [9]). Namely, if the modulus of the diagonal element $|\omega|$ in each row of the 
matrix $G_{n+1}(w ; \omega)$ is greater than the sum of remaining elements moduli of the same row, then $\operatorname{det} G_{n+1}(w ; \omega) \neq 0$. This implies the estimate

$$
\left|\omega_{w(n, 2 m)}^{ \pm}\right|<\Omega(n, 2 m):=\sum_{k=1}^{n} k^{2 m} .
$$

For example, for the second derivative $(m=1)$ we have

$$
\left|\omega_{w(n, 2)}^{ \pm}\right|<\Omega(n, 2)=\frac{1}{6} n(n+1)(2 n+1),
$$

while the calculations for $n=10$ give

$$
0.571 \cdot \Omega(10,2) \approx \omega_{w(10,2)}^{+}<\left|\omega_{w(10,2)}^{-}\right| \approx 0.666 \cdot \Omega(10,2) .
$$

As it can be easily checked, the growth order of $\Omega(n, 2 m)$ for $n \rightarrow \infty$ and each fixed $m$ has the form

$$
\Omega(n, 2 m) \asymp \frac{1}{2 m+1} n^{2 m+1}, \quad n \rightarrow \infty .
$$

The calculations up to $n=30$ show that this growth order is rather precise for $\left|\omega_{w}^{ \pm}(n, 2 m)\right|$. In particular, this is a distinctive property of the inequalities considered in Theorem 1 compared to the (sharp) classical inequalities $\left\|T_{n}^{(2 m)}\right\| \leqslant n^{2 m}\left\|T_{n}\right\|$ (S. N. Bernstein, M. Riesz [4], [15]).

\section{The application of AFT for estimating the derivative of} conjugate polynomials. The polynomial (1) with real coefficients can be represented as

$$
\begin{gathered}
T_{n}(t)=a_{0}+\sum_{k=1}^{n} \tau_{k}(t)=a_{0}+\operatorname{Re} \sum_{k=1}^{n}\left(a_{k}-i b_{k}\right) e^{i t k}, \\
\tau_{k}(t):=a_{k} \cos k t+b_{k} \sin k t .
\end{gathered}
$$

The polynomial

$$
\tilde{T}_{n}(t)=\sum_{k=1}^{n} a_{k} \sin k t-b_{k} \cos k t=\operatorname{Im} \sum_{k=1}^{n}\left(a_{k}-i b_{k}\right) e^{i t k}
$$

is called conjugate to the polynomial (1). It can be easily checked that $\tilde{T}_{n}(t)^{\prime}=\sum_{k=1}^{n} k \tau_{k}(t)$. Thus, for weights $w=w(n, 1)=\{1, \ldots, n\}$ we have from (2):

$$
\omega_{w}^{ \pm} a_{0}+\tilde{T}_{n}(t)^{\prime}=\omega_{w}^{ \pm} a_{0}+\mathcal{T}\left(T_{n}, w ; t\right)=\sum_{j=1}^{n} X_{j}^{ \pm} T_{n}\left(t-\lambda_{j}^{ \pm}\right),
$$


where $\omega_{w}^{ \pm}$are the largest and smallest roots of the Toeplitz determinant $\operatorname{det} G_{n+1}(w(n, 1) ; \omega)$, with $\sum_{j=1}^{n} X_{j}^{ \pm}=\omega_{w}^{ \pm}\left(\omega_{w}^{+}>0, \omega_{w}^{-}<0\right)$.

Similarly to Theorem 1, from the representation (11) we obtain

Theorem 2. For positive integer $n$, weights $w(n, 1):=\{1,2, \ldots, n\}$, $a_{0}=a_{0}\left(T_{n}\right)$ and all $t \in \mathbb{R}$, the following two-sided inequalities hold:

$$
\begin{aligned}
& \omega_{w}^{+}\left(\min _{x} T_{n}(x)-a_{0}\right) \leqslant \tilde{T}_{n}(t)^{\prime} \leqslant \omega_{w}^{+}\left(\max _{x} T_{n}(x)-a_{0}\right), \\
& \omega_{w}^{-}\left(\max _{x} T_{n}(x)-a_{0}\right) \leqslant \tilde{T}_{n}(t)^{\prime} \leqslant \omega_{w}^{-}\left(\min _{x} T_{n}(x)-a_{0}\right) .
\end{aligned}
$$

In particular, if $T_{n}$ is a positive-valued polynomial, then the first inequality in (12) and the second inequality in (13) imply

$$
-\omega_{w}^{+} a_{0} \leqslant \tilde{T}_{n}(t)^{\prime} \leqslant-\omega_{w}^{-} a_{0}, \quad a_{0}>0 .
$$

These inequalities are sharp, as there are extremal nonnegative polynomials $S_{ \pm}(t)$ of order $\leqslant n$ with the constant term $a_{0}\left(S_{ \pm}\right)>0$, for which the equalities hold:

$$
\omega_{w}^{ \pm} a_{0}\left(S_{ \pm}\right)+\tilde{S}_{ \pm}^{\prime}(0)=0 .
$$

Examples. Extremal polynomials are constructed as in the previous section, so we do not dwell on the details. For example, let $n=2$ and weights $w=\{1,2\}$; according to the formula (6), the following polynomials, for which the equalities (14) hold, are obtained:

$$
\begin{gathered}
S_{+}(t) \approx 0.125-0.125 \cos (2 t), \quad \omega_{w}^{+}=2 ; \\
S_{-}(t) \approx 0.158+0.125 \cos (2 t)+0.183 \cos (t), \quad \omega_{w}^{-} \approx-2.732 .
\end{gathered}
$$

For $n=3$ and $w=\{1,2,3\}$ we have

$$
\begin{gathered}
S_{+}(t) \approx 0.036-0.031 \cos (3 t)-0.025 \cos (2 t)+0.020 \cos (t) ; \\
S_{-}(t) \approx 0.047+0.031 \cos (3 t)+0.045 \cos (2 t)+0.061 \cos (t) ; \\
\omega_{w}^{+} \approx 3.414, \quad \omega_{w}^{-} \approx-5.162 .
\end{gathered}
$$

Remark 2. The estimate for $\omega_{w(n, 1)}^{ \pm}$by the above-mentioned Hadamard theorem gives:

$$
\left|\omega_{w(n, 1)}^{ \pm}\right|<\sum_{k=1}^{n} k=\frac{1}{2} n(n+1) .
$$


The calculations up to $n=30$ show that this growth order is rather precise. In particular, this is a distinctive property of the inequalities (12), (13) considered in Theorem 2 compared to the (sharp) classical Szegö inequalities $\left\|\tilde{T}_{n}^{\prime}\right\| \leqslant n\left\|T_{n}\right\|$, see [17].

Acknowledgments. The reported study was funded by RFBR according to the research project 20-31-90010.

\section{References}

[1] Arestov V. V. On extremal properties of the nonnegative trigonometric polynomials. Tr. Inst. Mat. Mekh., 1992, vol. 1, pp. 50-70.

[2] Beylkin G., Monzón L. On generalized gaussian quadratures for exponentials and their applications. Appl. Comput. Harmon. Anal., 2002, vol. 12, pp. 332 -373. DOI: https://doi.org/10.1006/acha.2002.0380

[3] Belov A. S. Some estimates for non-negative trigonometric polynomials and properties of these polynomials. Izv. Ross. Akad. Nauk Ser. Mat., 2003, vol. 67, no. 4, pp. 3-20. DOI: https://doi.org/10.4213/im440

[4] Bernstein S. N. Collected Works: vol. 2. The Constructive Theory of Functions. USSR Academy of Sciences Publishing House, 1954.

[5] Chunaev P., Danchenko V. Approximation by amplitude and frequency operators. J. Approx. Theory, 2016, vol. 207, pp. $1-31$.

DOI: https://doi.org/10.1016/j.jat.2016.02.005

[6] Danchenko V. I., Chkalova D. G. Algebraic analogs of Fejer inequalities. J. Math. Sci., 2021, vol. 255, no. 5, pp. 601-608.

[7] Danchenko V. I., Danchenko D. Ya. Extraction of pairs of harmonics from trigonometric polynomials by phase-amplitude operators. J. Math. Sci., 2018, vol. 232, no. 3, pp. $322-337$.

DOI: https://doi.org/10.1007/s10958-018-3875-0

[8] Egerváry E., Szász O. Some extremal problems in the field of trigonometric polynomials. Math. Z., 1928, vol. 27, no. 1, pp. 641-652.

[9] Gantmacher F. R. Theory of matrices. AMS Chelsea publishing, 1959.

[10] Gashkov S. B. The Fejer-Egervary-Szasz inequality for nonnegative trigonometric polynomials. Math. Prosvesh., 2005, vol. 9, pp. 69-75. (in Russian)

[11] Grenander U., Szegö G. Toeplitz forms and their applications. Chelsea Publishing Company, New York, 1984.

[12] Kalmykov S., Nagy B. Higher Markov and Bernstein inequalities and fast decreasing polynomials with prescribed zeros. J. Approx. Theory, 2018, vol. 226 , pp. $34-59$. 
[13] Pisarenko V. F. The retrieval of harmonics from a covariance function. Geophys. J. R. Astr. Soc., 1973, vol. 33, pp. 347-366.

[14] Polya G., Szego G. Problems and theorems in analysis. II - Theory of functions, zeros, polynomials, determinants, number theory, geometry. Springer-Verlag, Berlin, 1998.

[15] Riesz M. A trigonometric interpolation formula and some inequalities for polynomials. Deutsche Math. Ver, 1914, vol. 23, pp. 354-368.

[16] Stechkin S. B. Certain extremal properties of positive trigonometric polynomials. Mat. Zametki, 1970, vol. 7, pp. 411-422.

[17] Szegö G. Über einen Satz des Herrn Serge Bernstein. Schriften Königsberg, 1928 , vol. 5 , pp. $59-70$.

[18] Vasilchenkova D. G., Danchenko V. I. Extraction of harmonics from trigonometric polynomials by phase-amplitude operators. St. Petersburg Math. J., 2021. vol. 32. pp. 215-232.

DOI: https://doi.org/10.1090/spmj/1645

[19] Vasilchenkova D. G., Danchenko V. I. Extraction of several harmonics from trigonometric polynomials. Fejer-type inequalities. Proc. Steklov Inst. Math., 2020, vol. 308, pp. $92-106$.

DOI: https://doi.org/10.1134/S0081543820010083

Received April 25, 2021.

In revised form, August 03, 2021.

Accepted August 08, 2021.

Published online August 31, 2021.

Vladimir State University,

87, Gorky St., Vladimir 600000, Russia

V. I. Danchenko

vdanch2012@yandex.ru

D. G. Chkalova

darya.vasilchenkova@mail.ru 